(iii) The maximum bandwidth observed at a point in the diffracted wave field is $W_{m}=2 /(r \lambda)^{1 / 2}+2 \rho_{\max } \cos (\mathbf{k}, \mathbf{n}) / r \lambda$.

(iv) The wave field can be represented by the band-limited angular spectrum. When $\rho_{\max }<(r \lambda)^{1 / 2}$, the diffracted wave field can be approximately determined by superposing the angular spectrum of the object within the effective bandwidth $W=2 /(r \lambda)^{1 / 2}+\rho_{\max } \cos (\mathbf{k}, \mathbf{n}) / r \lambda$. When $\rho_{\max } \ll(r \lambda)^{1 / 2}$, it can be determined by superposing the angular spectrum within the effective bandwidth $W=2 /(r \lambda)^{1 / 2}$.

${ }^{1}$ C. J. Bouwkamp, "Diffraction theory,” Rep. Prog. Phys. 17, 35-100 (1954).

2 J. A. Ratcliffe "Some aspects of diffraction theory and their application to the ionosphere," Rep. Prog. Phys. 19, 188-267 (1956).

${ }^{3} \mathrm{G}$. C. Sherman "Diffracted wave fields expressible by plane-wave expansions containing only homogeneous waves," J. Opt. Soc. Am. 59, 697-711 (1969).

${ }^{4}$ G. C. Sherman, J. J. Stamnes, A. J. Devaney, and E. Lalor "Contribution of the inhomogeneous waves in angular-spectrum representations," Opt. Commun. 8, 271-274 (1973).

${ }^{5}$ W. H. Carter "Band-limited angular-spectrum approximation to a spherical scalar wave field," J. Opt. Soc. Am. 65, 1054-1058 (1975).
${ }^{6} \mathrm{E}$. T. Whittaker "On the partial differential equations of mathematical physics," Math. Ann. 57, 333-355 (1902).

${ }^{7}$ G. C. Sherman, A. J. Devaney, and L. Mandel "Plane-wave expansions of the optical field," Opt. Commun. 6, 115-118 (1972).

$8 \mathrm{~J}$. W. Goodman, Introduction to Furier Optics (McGraw-Hill, New York 1968) p. 61.

${ }^{9} \mathrm{H}$. M. Nussenzweig "Diffraction theory in the k-representation," An. Acad. Bras. Ciênc. 31, 515-521 (1959).

${ }^{10} Y$. Shono "Reconstructed images from volume holograms in the Fraunhofer approximations: Analysis by a new spherical-wave expansion," J. Opt. Soc. Am. 66, 564-574 (1976).

${ }^{11} \mathrm{H}$. Weyl "Ausbreitung elektromagnetischer Wellen über einen Leiter," Ann. Phys. (Paris) 60, 481-500 (1919).

${ }^{12} \mathrm{We}$ assume that a spherical wave is expanded to

$$
e^{i k r / i k r}=A \int_{0}^{2 \pi} \int_{0}^{\theta} e^{i \mathbf{k r}} \sin \theta d \theta d \varphi
$$

If there is no phase jump of the plane waves, $A$ must be a real constant. On this assumption, we get

$$
\theta=\cos ^{-1}\left(1-\frac{2 m+1}{2} \frac{\lambda}{r}\right), \quad m=0,1,2, \ldots
$$

Therefore the minimum angle becomes $\theta_{1}$ given by (4).

${ }^{13} \mathrm{~F}$. A. Jenkins and H. E. White, Fundamentals of Optics, 3rd ed. (McGraw-Hill, New York, 1957) p. 374.

\title{
Modal dispersion in lightguides in the presence of strong coupling
}

\author{
Bruno Crosignani* and Charles H. Papas \\ California Institute of Technology, Pasadena, California 91125 \\ Paolo Di Porto \\ Fondazione Ugo Bordoni, Istituto Superiore P. T., Viale Europa, Roma, Italy \\ (Received 31 January 1978)
}

\begin{abstract}
The effect of strong mode coupling on modal dispersion in optical fibers has been investigated. The pulse dispersion turns out to be qualitatively different from the one relative to the weak-coupling case, while it exhibits a drastic reduction as compared with that of the uncoupled case. The role of the initial pulse length and of the source coherence time has been elucidated.
\end{abstract}

\section{INTRODUCTION}

The role of mode coupling in the propagation of guided modes in a multimode lightguide is well established. It can be negative or positive, according whether the purpose is to let the lower-order modes propagate without progressively sharing their energy with the higher-order ones, or to reduce the modal dispersion of an optical fiber. While the first statement can be easily understood, the second one is not so obvious and has to be proved analytically. In fact, the existing theory ${ }^{1,2}$ is based on a statistical approach and it describes the propagation of the average power in the $m$ th mode $\left\langle P_{m}(z, t)\right\rangle$, where the averaging operation $\langle\ldots .$.$\rangle is meant to be per-$ formed over an ensemble of many macroscopically equivalent lightguides differing among themselves because of microscopically random imperfections.

In this frame it can be shown that, under suitable assumptions on the coupling, the centers of mass of the wave packets describing the $\left\langle P_{m}(z, t)\right\rangle$ 's tend to travel with a common average velocity, while the pulse widths turn out to be proportional to $(a+b z)^{1 / 2}, a$ and $b$ being two positive constants, and $z$ the traveled distance. This fact is interpreted as a mechanism of reduction of the modal dispersion in optical fibers, ${ }^{1,2}$ since one would expect, should the modes travel independently as they do in the absence of mode coupling, a distortion proportional to the trayeled distance $z$.

The main limitation of the statistical approach is connected with the presence of fluctuations around the average value, which, if too ample, do not allow the average result to be confidently applied to the single fiber-with which one most often deals in practical situations. It has been demonstrated ${ }^{3}$ that in the case in which the fiber is excited by a polychromatic source there is a fiber length $L \propto 1 / T_{c}$ ( $T_{c}$ being the coherence time of the source) such that the fluctuations of the energy per mode $I_{m}(z, t)$ :

$$
I_{m}(z, t)=\int_{-\infty}^{\infty} P_{m}(z, t) d t
$$

tend to vanish for $z$ larger than $L$. However, this result does not concern the behavior of $P_{m}(z, t)$ itself, but for the stationary case in which $P_{m}(z, t)$ is time independent. Besides, the statistical approach does not give a correct result for the single fiber when one has to evaluate the cross-correlation term between the field $\mathbf{e}_{m}(\mathbf{r}, z, t)$ and $\mathbf{e}_{n}(\mathbf{r}, z, t)$ pertaining to the $m$ th and $n$th mode, that is 


$$
T_{n m}=\left\langle\mathbf{e}_{m}(\mathbf{r}, z, t) \cdot \mathbf{e}_{n}^{*}(\mathbf{r}, z, t)\right\rangle_{\mathrm{av}},
$$

where the symbol $\langle\ldots \ldots\rangle_{\text {av }}$ indicates the averaging operation over the fluctuations of the source exciting the lightguide. As a matter of fact, it has been shown ${ }^{3}$ that $\left\langle T_{n m}\right\rangle$ goes to zero over a distance not larger than $T_{c}\left|1 / V_{n}-1 / V_{m}\right|^{-1}\left(V_{n}\right.$ and $V_{m}$ being the $n$th and $m$ th mode group-velocity), which is just the distance over which it would go to zero in the absence of mode coupling. ${ }^{4}$ This obviously cannot be the case for the single fiber, since the presence of a coupling certainly gives rise to a correlation between the modes over a longer distance.

The previous considerations make clear that it would be desirable to be able to find, at least for some simple workable models, an analytic solution for the problem of propagation in a deterministic case (that is without resorting to the statistical approach), in order to compare it with the above mentioned results. This would also allow us to evaluate in a correct way the $T_{n m}$ 's, whose behavior may furnish a simple way of gaining information on dispersion - and thus on mode coupling. ${ }^{5}$ In this paper, this program is carried out introducing the hypothesis of strong coupling, a case interesting per se which cannot be investigated by means of the statistical treatment which covers weak coupling, and considering a simple model in which only two modes interact.

In the framework of our approach, strong coupling means that the magnitude of the coupling constant $K_{12}$ is much larger than the difference $\left|\beta_{1}-\beta_{2}\right|$ between the propagation constants of the two unperturbed modes [see Eq. (24)]. Under this assumption, we consider the situation of resonant coupling, for which the characteristic spatial periodicity $l$ of $K_{12}$ if of the order of $\left|\beta_{1}-\beta_{2}\right|^{-1}$, and that of slowly varying coupling, for which $l \gg\left|\beta_{1}-\beta_{2}\right|^{-1}$. In both cases, propagation is significantly affected by the fact that $\left|K_{12}\right|$ is large (while, as is well known, the fulfilment of the resonant condition is essential in the weak-coupling regime ${ }^{2}$ ). Dispersion turns out to be drastically reduced with respect to the case in which coupling is absent, and its qualitative behavior differs from what one would expect according to the statitistical method, in that the pulse spreading turns out to be proportional to the fiber length $z$ instead that to $z^{1 / 2}$.

The analysis of the propagation in the presence of strong coupling, besides allowing us to complete the description of the effects of mode coupling on modal dispersion, can be relevant for the study of propagation in mode scramblers, which are strongly-coupling fiber samples able to excite all guided modes in a repeatable manner. ${ }^{6}$

\section{DESCRIPTION OF PROPAGATION IN LIGHTGUIDES}

The transverse electromagnetic field propagating in a cylindrical guiding structure can be expressed as the superposition of the fields pertaining to each guided mode in the form $^{3,7}$

$$
\mathbf{E}(\mathbf{r}, z, t)=\sum_{m} \mathbf{e}_{m}(\mathbf{r}, z, t),
$$

with

$$
\mathbf{e}_{m}(\mathbf{r}, z, t)=\mathbf{E}_{m}(\mathbf{r}) a_{m}(z, t),
$$

where the $\mathbf{E}_{m}(\mathbf{r})$ 's represent the modes of the ideal guiding structure (that is without mode coupling) and the expansion coefficients $a_{m}(z, t)$ are defined through the relation

$$
a_{m}(z, t)=\int_{-\infty}^{\infty} c_{m}(z, \omega) e^{i \omega t-i \beta_{m}(\omega) z} d \omega .
$$

In Eq. (5), $\beta_{m}(\omega)$ is the propagation constant of the $m$ th mode and the $c_{m}$ 's depend on $z$ because of the presence of mode coupling [otherwise one would have $c_{m}(z, \omega)=c_{m}(0, \omega)$ ].

It is possible to show ${ }^{6}$ that as long as only forward traveling modes are considered the transverse part of the magnetic field obeys equations identical to Eqs. (3) and (4), provided the substitution $\mathbf{E}_{m} \rightarrow \mathbf{H}_{m}$ is made. From the above considerations it follows that, in order to evaluate second-order averages of the kind

$$
G\left(\mathbf{r}_{1}, \mathbf{r}_{2}, t_{1}, t_{2}, z\right)=\left\langle\mathbf{E}\left(\mathbf{r}_{1}, z, t_{1}\right) \cdot \mathbf{E}^{*}\left(\mathbf{r}_{2}, z, t_{2}\right)\right\rangle_{\mathrm{av}},
$$

or

$$
P^{\sigma}(z, t)=\frac{1}{2} \operatorname{Re} \int_{\sigma} d \mathbf{r}\left\langle\mathbf{E}(\mathbf{r}, z, t) \times \mathbf{H}^{*}(\mathbf{r}, z, t)\right\rangle_{\mathrm{av}} \cdot \mathbf{e}_{z}
$$

( $\mathbf{e}_{z}$ being a unit vector in the $z$ direction), representing, respectively, the mutual coherence function and the power carried by the electromagnetic field through a given area $\sigma$ of a fiber section, it is sufficient to investigate the behavior of the quantities

$$
\left\langle a_{m}\left(z, t_{1}\right) a_{n}^{*}\left(z, t_{2}\right)\right\rangle_{\mathrm{av}}
$$

or, equivalently, of

$$
\left\langle c_{m}(z, \omega) c_{n}^{*}\left(z, \omega^{\prime}\right)\right\rangle_{\mathrm{av}} .
$$

According to Eqs. (3), (4), and (7) and the mode orthogonality, it turns out ${ }^{3}$ that $\left\langle a_{m}(z, t) a_{m}^{*}(z, t)\right\rangle_{\text {av }}$ is proportional to the power $P_{m}(z, t)$ carried by the $m$ th mode through the whole fiber section, and that

$$
P^{\infty}(z, t)=\sum_{m} P_{m}(z, t),
$$

while the nondiagonal terms $\left\langle a_{m}(z, t) a_{n}^{*}(z, t)\right\rangle_{\text {av }}$ can be connected with the degree of correlation between the various propagating modes. In particular one has

$$
\begin{aligned}
P^{\sigma}(z, t)=\frac{1}{2} \operatorname{Re} \sum_{m} \sum_{n} F_{m n}^{\sigma}\left\langle a_{m}(z, t) a_{n}^{*}(z, t)\right\rangle_{\mathrm{av}} \\
=\operatorname{Re} \sum_{m}\left(F_{m m}^{\sigma} / F_{m m}^{\infty}\right) P_{m}(z, t) \\
+\frac{1}{2} \operatorname{Re} \sum_{m \neq n} \sum_{m n} F_{m}^{\sigma}\left\langle a_{m}(z, t) a_{n}^{*}(z, t)\right\rangle_{\mathrm{av}}
\end{aligned}
$$

with

$$
F_{m n}^{\sigma}=\int_{\sigma} d \mathbf{r} \mathbf{E}_{m}(r) \times \mathbf{H}_{n}^{*}(\mathbf{r}) \cdot \mathbf{e}_{z},
$$

showing that the nondiagonal terms represent interference contributions between the various modes, which are always present whenever $\sigma$ is finite (otherwise they disappear due to the orthogonality between different modes). 'Thus, the analysis of $P^{\sigma}(z, t)$, as compared with $P^{\infty}(z, t)$, can furnish a way of estimating the degree of correlation between the propagating modes.

In the absence of mode coupling, one has $c_{m}(z, \omega)=c_{m}(0, \omega)$, which, together with the approximate relation

$$
\beta_{m}(\omega)=\beta_{m}\left(\omega_{0}\right)+\left(\omega-\omega_{0}\right) / V_{m}
$$


(which implies, as a necessary condition, the ratio between the bandwidth $\delta \omega$ of the propagating signal and the central frequency $\omega_{0}$ to be much less than one), furnishes [see Eq. (5)]

$$
a_{m}(z, t)=a_{m}\left(0, t-z / V_{m}\right),
$$

where $V_{m}=\left(d \beta_{m} / d \omega\right)_{\omega=\omega_{0}}^{-1}$ is the group velocity of the $m$ th mode. This amounts to saying that $P_{m}(z, t)$ propagates with this velocity and that the nondiagonal terms

$$
\begin{aligned}
\left\langle a_{m}(z, t) a_{n}^{*}(z, t)\right\rangle_{\mathrm{av}} & \\
& =\left\langle a_{m}\left(0, t-\bar{z} / V_{m}\right) a_{n}^{*}\left(0, t-z / V_{n}\right)\right\rangle_{\mathrm{av}}
\end{aligned}
$$

vanish whenever the fiber length is such that they acquire a time delay larger than the coherence time $T_{c}=2 \pi / \delta \omega$ of the source; that is,

$$
\left|z / V_{m}-z / V_{n}\right| \geq T_{\mathrm{c}}
$$

\section{PROPAGATION IN THE PRESENCE OF MODE COUPLING: NONRESONANT CASE}

In order to have an analytically solvable model, we consider a simple case in which only two modes 1 and 2 are interacting. The evolution with $z$ of the mode amplitudes $c_{1}(z, \omega)$ and $c_{2}(z, \omega)$ is described by the following set of equations ${ }^{8}$ :

$$
\begin{gathered}
\frac{d c_{1}(z, \omega)}{d z}=K_{12}(z) e^{i \Delta(\omega) z} c_{2}(z, \omega), \\
\frac{d c_{2}(z, \omega)}{d z}=-K_{12}^{*}(z) e^{-i \Delta(\omega) z} c_{1}(z, \omega),
\end{gathered}
$$

where $\Delta(\omega)=\beta_{1}(\omega)-\beta_{2}(\omega), K_{12}(z)$ is a $z$-dependent coupling coefficient and the self-coupling coefficients $K_{11}(z), K_{22}(z)$, which would not give rise to any relevant effect, have been omitted for the sake of simplicity. By means of the change of dependent variables

$$
\begin{aligned}
& c_{1}(z, \omega)=b_{1}(z, \omega) e^{i \Delta(\omega) z / 2} \\
& c_{2}(z, \omega)=b_{2}(z, \omega) e^{-i \Delta(\omega) z / 2}
\end{aligned}
$$

one gets from Eq. (17),

$$
\begin{gathered}
\frac{d b_{1}}{d z}+i(\Delta / 2) b_{1}=K_{12} b_{2} \\
\frac{d b_{2}}{d z}-i(\Delta / 2) b_{2}=-K_{12}^{*} b_{1}
\end{gathered}
$$

from which, by eliminating $b_{2}$, one arrives at

$$
\begin{aligned}
b_{1}^{\prime \prime}-\left(K_{12}^{\prime} / K_{12}\right) b_{1}^{\prime}+\left[\left|K_{12}\right|^{2}+\right. & \Delta^{2} / 4 \\
& \left.-i\left(K_{12}^{\prime} / 2 K_{12}\right) \Delta\right] b_{1}=0,
\end{aligned}
$$

where the prime indicates differentiation with respect to $z$. By performing the substitution

$$
b_{1}=\tilde{b}_{1} \exp \left(\frac{1}{2} \int^{z}\left(K_{12}^{\prime} / K_{12}\right) d z\right),
$$

one finally obtains

$$
\begin{aligned}
\tilde{b}_{1}^{\prime \prime}+\left[\left|K_{12}\right|^{2}+\Delta^{2}\right. & =i\left(K_{12}^{\prime} / 2 K_{12}\right) \Delta \\
& \left.+\left(K_{12}^{\prime} / 2 K_{12}\right)^{\prime}-\left(K_{12}^{\prime} / 2 K_{12}\right)^{2}\right] \tilde{b}_{1}=0 .
\end{aligned}
$$

Let us now assume $K_{12}(z)$ to be a slowly varying function of $z$. This means that there is no Fourier component of the coupling process that would provide coupling in the sense of first-order perturbation theory. However, this fact does not preclude an effective coupling, with a consequent influence on dispersion, for $\left|K_{12}\right|$ large enough, as the following derivation will make clear. The last three terms inside the square bracket in Eq. (22) can be neglected, as compared with $\left|K_{12}\right|^{2}$ $+\Delta^{2} / 4$, provided that the condition $l \gg|1 / \Delta|$ is fulfilled, $l$ being a characteristic oscillation length of $K_{12}(z)$. The resulting equation can be easily solved in the WKB approximation, under the usual adiabatic hypothesis

$$
\frac{\frac{d}{d z}\left[\left(\left|K_{12}\right|^{2}+\Delta^{2} / 4\right)^{1 / 2}\right]}{\left|K_{12}\right|^{2}+\Delta^{2} / 4} \ll 1 .
$$

In particular, in the strong-coupling regime,

$$
\left|K_{12}\right|^{2} \gg \Delta^{2}
$$

and by assuming, for the sake of simplicity and without loss of validity of the main conclusions of our derivation, $K_{12}$ to be independent from $Z$, one obtains

$$
\begin{aligned}
a_{1}(z, t)=\int d \omega \exp \left\{-i\left[\beta_{1}(\omega)+\beta_{2}(\omega)\right] z / 2+i \omega t\right\} \\
\times\left[A \exp \left(i\left|K_{12}\right| z+i \Delta^{2} z / 8\left|K_{12}\right|\right)\right. \\
\left.\quad+B \exp \left(-i\left|K_{12}\right| z-i \Delta^{2} z / 8\left|K_{12}\right|\right)\right] \\
a_{2}(z, t)=i \exp (-i \phi) \int d \omega \exp \left\{-i\left[\beta_{1}(\omega)+\beta_{2}(\omega)\right] z / 2+i \omega t\right\} \\
\times\left[A \exp \left(i\left|K_{12}\right| z+i \Delta^{2} z / 8\left|K_{12}\right|\right)\right. \\
\left.\quad-B \exp \left(-i\left|K_{12}\right| z-i \Delta^{2} z / 8\left|K_{12}\right|\right)\right]
\end{aligned}
$$

with

$$
\begin{aligned}
& A=(1 / 2)\left[c_{1}(0, \omega)-i e^{i \phi} c_{2}(0, \omega)\right], \\
& B=(1 / 2)\left[c_{1}(0, \omega)+i e^{i \phi} c_{2}(0, \omega)\right],
\end{aligned}
$$

and $\exp (i \phi)=K_{12} /\left|K_{12}\right|$. Assuming $c_{1}(0, \omega)$ and $c_{2}(0, \omega)$ to possess the same $\omega$ dependence, the problem is reduced to the investigation of the quantities

$$
\begin{aligned}
J_{a}(z, t)=\int d \omega & c_{1}(0, \omega) \exp \left\{-i\left[\beta_{1}(\omega)+\beta_{2}(\omega)\right] z / 2\right\} \\
& \times \exp \left[i \omega t+i\left|K_{12}\right| z+i \Delta^{2}(\omega) z / 8\left|K_{12}\right|\right],
\end{aligned}
$$

and

$$
\begin{aligned}
J_{b}(z, t)=\int & d \omega c_{1}(0, \omega) \exp \left\{-i\left[\beta_{1}(\omega)+\beta_{2}(\omega)\right] z / 2\right\} \\
& \times \exp \left[i \omega t-i\left|K_{12}\right| z-i \Delta^{2}(\omega) z / 8\left|K_{12}\right|\right],
\end{aligned}
$$

which is obtained from $J_{1}$ by means of the substitution $\left|K_{12}\right|$ $\rightarrow-\left|K_{12}\right|$.

By taking advantage of Eq. (13), one can write

$$
\Delta(\omega)=\Delta\left(\omega_{0}\right)+\left(\omega-\omega_{0}\right) / V^{(-)}
$$

where

$$
1 / V^{(-)}=1 / V_{1}-1 / V_{2},
$$

which, once introduced in Eq. (29), yields

$$
\left.J_{b}(z, t)=e^{i \psi} \int d \omega c_{1}(0, \omega) e^{-i \omega^{2} \tau^{2}} e^{i \omega(t}-z / \tilde{v}_{b}\right)
$$

with

$$
\begin{array}{r}
\psi=-\left[\beta_{1}\left(\omega_{0}\right)+\beta_{2}\left(\omega_{0}\right)\right] z / 2+\omega_{0} z / 2 V^{(+)}-\left|K_{12}\right| z \\
-\Delta^{2}\left(\omega_{0}\right) z / 8\left|K_{12}\right|+\Delta\left(\omega_{0}\right) \omega_{0} z / 4 V^{(-)}\left|K_{12}\right| \\
-\omega_{0}^{2} z / 8 V^{(-)^{2}}\left|K_{12}\right|
\end{array}
$$

and

$$
\tau^{2}=z / 8 V^{(-) 2}\left|K_{12}\right|
$$


having introduced the new velocities $V^{(+)}$and $\tilde{v}_{b}$ defined by the relations

$$
\begin{gathered}
1 / V^{(+)}=1 / V_{1}+1 / V_{2}, \\
1 / \hat{v}_{b}=1 / 2 V^{(+)}+\Delta\left(\omega_{0}\right) / 4 V^{(-)}\left|K_{12}\right|-\omega_{0} / 4 V^{(-)^{2}}\left|K_{12}\right| .
\end{gathered}
$$

An analogous expression holds for $J_{a}(z, t)$ once the substitution $\left|K_{12}\right| \rightarrow-\left|K_{12}\right|$ is performed, which in particular defines another velocity

$$
1 / \tilde{v}_{a}=1 / 2 V^{(+)}-\Delta\left(\omega_{0}\right) / 4 V^{(-)}\left|K_{12}\right|+\omega_{0} / 4 V^{(-)^{2}}\left|K_{12}\right| .
$$

We have now to consider the quantities $J_{a} J_{a}^{*}, J_{b} J_{b}^{*}$, and $J_{a} J_{b}^{*}$, and to average them over the fluctuations of the source (which, for a stationary source, is equivalent to taking the time average over an interval of the order of the coherence time $T_{c}$ ). In fact, their linear combinations furnish the significant quantities $\left\langle\left|a_{1}(z, t)\right|^{2}\right\rangle_{\mathrm{av}},\left\langle\left|a_{2}(z, t)\right|^{2}\right\rangle_{\mathrm{av}}$ and $\left\langle a_{1}(z, t) a_{2}^{*}(z, t)\right\rangle_{\mathrm{av}}$. Thus, according to Eq. (32), one has to evaluate the quantity

$$
\left\langle c_{1}(0, \omega) c_{1}^{*}\left(0, \omega^{\prime}\right)\right\rangle_{\mathrm{av}} .
$$

This can be done by assuming the electric field at $z=0$ to possess a temporal behavior of the kind

$$
e^{i \omega_{0} t} F(t) S(t)
$$

where $F(t)$ is a rapidly varying function accounting for the source bandwidth while $S(t)$ represents the (usually) much slower amplitude modulation of the carrier. By assuming

$$
S(t) \propto \exp \left(-t^{2} / T_{p}^{2}\right)
$$

and

$$
\left\langle F\left(t^{\prime}\right) F^{*}\left(t^{\prime \prime}\right)\right\rangle_{\mathrm{av}} \propto \exp \left[-\left(t^{\prime}-t^{\prime \prime}\right)^{2} / T_{c}^{2}\right],
$$

where the subscripts $p$ and $c$ stand, respectively, for pulse and coherence, one has (see Appendix)

$$
\begin{aligned}
& \left\langle c_{1}(0, \omega) c_{1}^{*}\left(0, \omega^{\prime}\right)\right\rangle_{\mathrm{av}} \\
& \quad \propto \exp \left(-\Omega^{2} T_{1}^{2} / 4-\Omega^{\prime 2} T_{1}^{2} / 4+\Omega \Omega^{\prime} T_{2}^{2} / 2\right),
\end{aligned}
$$

where

$$
T_{1}^{2}=T_{p}^{2}\left(T_{p}^{2}+T_{\mathrm{c}}^{2}\right) /\left(2 T_{p}^{2}+T_{c}^{2}\right)
$$

and

$$
T_{2}^{2}=T_{p}^{4} /\left(2 T_{p}^{2}+T_{c}^{2}\right)
$$

with $\Omega=\omega-\omega_{0}$ and $\Omega^{\prime}=\omega^{\prime}-\omega_{0}$. In particular, the monochromatic and the stationary cases are respectively recovered by letting $T_{c}$ and $T_{p}$ become infinitely large.

By taking advantage of Eq. (42), one can write

$$
\begin{aligned}
\left\langle J_{b}(z, t) J_{b}^{*}(z, t)\right\rangle_{\mathrm{av}} \propto \int d \omega \int & d \omega^{\prime} \exp \left[-\omega^{2}\left(i \tau^{2}+T_{1}^{2} / 4\right)-\omega^{\prime 2}\left(-i \tau^{2}+T_{1}^{2} / 4\right)\right] \\
& \times \exp \left\{\omega\left[i t_{b}+\omega_{0}\left(T_{1}^{2}-T_{2}^{2}\right) / 2\right]+\omega^{\prime}\left[-i t_{b}+\omega_{0}\left(T_{1}^{2}-T_{2}^{2}\right) / 2+\omega T_{2}^{2} / 2\right]\right\} \exp \left[-\omega_{0}^{2}\left(T_{1}^{2}-T_{2}^{2}\right) / 2\right]
\end{aligned}
$$

with $t_{b}=t-z / \tilde{v}_{b}$. After performing the two integrals over $\omega$ and $\omega^{\prime}$, Eq. (45) furnishes

$$
\left\langle J_{b} J_{b}^{*}\right\rangle_{\mathrm{av}} \propto(1 / T) \exp \left[-\left(t-z / v_{b}\right)^{2} / T^{2}\right]
$$

where

$$
\begin{gathered}
T=\left(T_{0}^{2} / 2+8 \tau^{4} / T_{3}^{2}\right)^{1 / 2}, \\
1 / v_{b}=1 / 2 V^{(+)}+\Delta\left(\omega_{0}\right) / 4 V^{(-)}\left|K_{12}\right|
\end{gathered}
$$

with

$$
\begin{gathered}
T_{3}^{2}=T_{1}^{2}-T_{2}^{2}=T_{p}^{2} T_{c}^{2} /\left(2 T_{p}^{2}+T_{c}^{2}\right), \\
T_{0}^{2}=T_{1}^{2}+T_{2}^{2}=\left(2 T_{p}^{4}+T_{p}^{2} T_{c}^{2}\right) /\left(2 T_{p}^{2}+T_{c}^{2}\right) .
\end{gathered}
$$

An.analogous expression holds true for $\left\langle J_{a} J_{a}^{*}\right\rangle_{\mathrm{av}}$, which reads

$$
\left\langle J_{a} J_{a}^{*}\right\rangle_{a v} \propto(1 / T) \exp \left[-\left(t-z / v_{a}\right)^{2} / T^{2}\right]
$$

where

$$
1 / v_{a}=1 / 2 V^{(+)}-\Delta\left(\omega_{0}\right) / 4 V^{(-)}\left|K_{12}\right|
$$

One can observe that the group-velocities $v_{a}$ and $v_{b}$ could have been also directly obtained by taking, respectively, the derivative with respect to $\omega$ (evaluated at $\omega=\omega_{0}$ ) of the propagation constants of the first and second terms contributing to $a_{1}$ [Eq. (25)] or $\mathrm{a}_{2}$ [Eq. (26)].

The term $\left\langle J_{a} J_{b}^{*}\right\rangle_{\text {av }}$ cannot be expressed in the form of a wave packet propagating with a definite velocity, and will be discussed later on.
The expression of $\left\langle J_{a} J_{a}^{*}\right\rangle_{\text {av }}$ and $\left\langle J_{b} J_{b}^{*}\right\rangle_{\text {av }}$, as given by Eqs. (46) and (51), shows that the signal inside the fiber evolves in two distinct pulses, traveling with two slightly different velocities $v_{a}$ and $v_{b}$. Besides, the temporal width of each pulse increases with $z$, so that one has to take into account two different sources of dispersion. In practice, this second kind of dispersion can be neglected for the values of $z$ such that [see Eq. (47)]

$$
T^{2}(z) / T_{3}<T_{0} / 4 \simeq T_{p} / 4
$$

The other contribution can be put in a quantitative form by introducing the time delay $T_{d}$ between the centers of mass of the two pulses at a distance $z$; that is,

$$
T_{d}(z)=z\left(1 / v_{b}-1 / v_{a}\right)=\Delta\left(\omega_{0}\right) z / 2 V^{(-)}\left|K_{12}\right| .
$$

It is immediate to see that this kind of dispersion is dominant-the distance between the centers of mass of the two pulses increasing more rapidly than their widths. In fact, it follows from Eqs. (34) and (54) that

$$
\begin{aligned}
& T_{d} T_{3} / \tau^{2}=4 V^{(-)} \Delta\left(\omega_{0}\right) T_{3} \\
& =4 \omega_{0} T_{3} V^{(-)} \Delta\left(\omega_{0}\right) / \omega_{0} \simeq 4 \omega_{0} T_{3},
\end{aligned}
$$

having taken into account the circumstance that the difference between the inverse of group and phase velocities, $V^{(-)}$and $\omega_{0} / \Delta\left(\omega_{0}\right)$, are of the same order. ${ }^{9}$ Observing that $T_{3}$ is of the order of the coherence time, the ratio between $T_{d}$ and $\tau^{2} / T_{3}$ turns out to be $\omega_{0} / \delta \omega \gg 1$. 
Returning now to the problem of evaluating the influence of the term $\left\langle J_{a} J_{b}^{*}\right\rangle_{a v}$, it may be noted that its importance tends to become negligible for distances such that

$$
T_{d}(z)>T_{p},
$$

after which the two pulses do not overlap anymore.

\section{PROPAGATION IN THE PRESENCE OF MODE COUPLING: RESONANT CASE}

The resonant case corresponds to a sinusoidally modulated coupling constant

$$
K_{12}(z)=2 K \cos (\chi z)
$$

with

$$
\chi=\Delta\left(\omega_{0}\right),
$$

the (resonance) frequency $\omega_{0}$ having been assumed to coincide with the central frequency of the exciting source. By introducing Eq. (57) into Eqs. (17) one obtains

$$
\begin{gathered}
\frac{d c_{1}(z, \omega)}{d z}=K e^{-i \chi z+i \Delta(\omega) z} c_{2}(z, \omega), \\
\frac{d c_{2}(z, \omega)}{d z}=-K^{*} e^{i \chi z-i \Delta(\omega) z} c_{1}(z, \omega),
\end{gathered}
$$

having neglected in the right-hand side of Eqs. (59) the terms containing the rapidly oscillating factors $\exp \left\{ \pm i\left[\Delta\left(\omega_{0}\right)+\chi\right] z\right\}$ with respect to the slowly varying terms containing $\exp \left\{ \pm i\left[\Delta\left(\omega_{0}\right)-\chi\right] z\right\}$, whenever $|K| \ll|x+\Delta(\omega)|$. By using this approximation, it is possible to write

$$
K_{12}=K e^{-i \Delta\left(\omega_{0}\right) z} .
$$

One can now use the procedure of Sec. III, thus obtaining

$$
\begin{aligned}
& a_{1}(z, t)= \exp \left(\frac{-i \Delta\left(\omega_{0}\right) z}{2}\right) \int d \omega \\
& \times \exp \left(-i \frac{\beta_{1}(\omega)+\beta_{2}(\omega)}{2} z+i \omega t\right) \\
& \times {\left[A \operatorname { e x p } \left(i|K| z+\frac{i \Delta^{2}(\omega) z}{8|K|}-\frac{i \Delta\left(\omega_{0}\right) \Delta(\omega) z}{4|K|}\right.\right.} \\
&+\left.\frac{i \Delta^{2}\left(\omega_{0}\right) z}{8|K|}\right)+B \exp \left(-i|K| z-\frac{i \Delta^{2}(\omega) z}{8|K|}\right. \\
&\left.+\frac{i \Delta\left(\omega_{0}\right) \Delta(\omega) z}{4|K|}-\frac{i \Delta^{2}\left(\omega_{0}\right) z}{8|K|}\right) \quad(61) \\
& a_{2}(z, t)= i \exp \left(-i \Phi+\frac{i \Delta\left(\omega_{0}\right) z}{2}\right) \int d \omega \\
& \times \exp \left(-i \frac{\beta_{1}(\omega)+\beta_{2}(\omega)}{2} z+i \omega t\right) \\
& \times\left[A \exp \left(i|K| z+\frac{i \Delta^{2}(\omega) z}{8|K|}-\frac{i \Delta\left(\omega_{0}\right) \Delta(\omega) z}{4|K|}+\frac{i \Delta^{2}\left(\omega_{0}\right) z}{8|K|}\right)\right. \\
&\left.-B \exp \left(-i|K| z-\frac{i \Delta^{2}(\omega) z}{8|K|}+\frac{i \Delta\left(\omega_{0}\right) \Delta(\omega) z}{4|K|}-\frac{i \Delta^{2}\left(\omega_{0}\right) z}{8|K|}\right)\right],
\end{aligned}
$$

with

$$
\begin{aligned}
& A=(1 / 2)\left[c_{1}(0, \omega)-i e^{i \Phi} c_{2}(0, \omega)\right], \\
& B=(1 / 2)\left[c_{1}(0, \omega)+i e^{i \Phi} c_{2}(0, \omega)\right],
\end{aligned}
$$

where $\exp (i \Phi)=K /|K|$.

The expressions of $a_{1}(z, t)$ and $a_{2}(z, t)$ show that the main properties of propagation can be deduced following the derivation of Sec. III. In the present resonant case, the groupvelocities $v_{a}$ and $v_{b}$ of both contributions to $a_{1}$ (and $a_{2}$ ) coincide, since

$$
\begin{array}{r}
\frac{1}{v_{a, b}}=\frac{d}{d \omega}\left(\frac{\beta_{1}(\omega)+\beta_{2}(\omega)}{2} \mp \frac{\Delta^{2}(\omega)}{8|K|} \pm \frac{\Delta\left(\omega_{0}\right) \Delta(\omega)}{4|K|}\right)_{\omega=\omega_{0}} \\
=\frac{1}{2}\left(\frac{1}{V_{1}}+\frac{1}{V_{2}}\right),
\end{array}
$$

so that the signal appears in the form of a single pulse undergoing a temporal broadening $T$ given by Eq. (47), which for large $z$ reduces to

$$
T=8^{1 / 2} \frac{\tau^{2}}{T_{3}}=\frac{1}{8^{1 / 2}} \frac{z}{V^{(-)^{2}}\left|K_{12}\right| T_{3}} .
$$

In other words, the two distinct pulses of the nonresonant situation overlap, so that the only cause of dispersion is given by their common broadening.

\section{INFLUENCE OF MODE COUPLING ON DISPERSION}

One can compare the results of the previous sections with the one obtained in the frame of the statistical-coupled power theory, according to which the temporal width of the pulse $T_{s}$ is proportional to the square root of $z$, that is ${ }^{10}$

$$
T_{s}=(2 z)^{1 / 2} / V^{(-)} h^{1 / 2},
$$

where

$$
h=\int_{-\infty}^{\infty} d \zeta\left\langle K_{12}(\zeta) K_{12}^{*}(0)\right\rangle e^{i \Delta\left(\omega_{0}\right) \zeta},
$$

the angular brackets indicating the averaging operation over an ensemble of many macroscopically similar fibers. Equations (54), (65), and (66) show that the results of the weak- and strong-coupling cases are qualitatively different. In particular, the deterministic approach brings under consideration the role of the coherence time $T_{c}$ of the exciting source and of the pulse duration $T_{p}$. It provides a dispersion which exhibits the same linear $z$ dependence of the uncoupled case, for which the width $T_{u}$ of the signal carried by the two uncoupled modes is given by ${ }^{10}$

$$
T_{u}=z / V^{(-)},
$$

but which is quantitatively very different. In fact, one has,

$$
T_{d} / T_{u}=\Delta\left(\omega_{0}\right) / 2\left|K_{12}\right| \ll 1
$$

and

$$
T / T_{u} \ll T_{d} / T_{u}=\Delta\left(\omega_{0}\right) / 2\left|K_{12}\right| \ll 1,
$$

where the first inequality in Eq. (70) follows from Eq. (55), in agreement with the intuitive statement that the presence of a strong coupling must considerably reduce dispersion, in both resonant and nonresonant situations.

We wish to stress again the fact that the present deterministic method deals with a case in which the $c_{m}$ 's considerably vary, in the nonresonant case, over a distance of the order $1 / \Delta\left(\omega_{0}\right)$ (strong coupling), while the opposite hypothesis is introduced in order to deal with the statistical approach. ${ }^{3}$ 
Actually, the strong-coupling hypothesis has as a consequence that the powers per mode $P_{1}(z, t)$ and $P_{2}(z, t)$ possess the same temporal evolution. Accordingly, two initially correlated modes travel without acquiring any mutual time delay, so that $\left\langle a_{1}(z, t) a_{2}^{*}(z, t)\right\rangle_{\mathrm{av}}$ does not vanish after the distance defined by Eq. (16), which implies that the transverse spatial correlation between them is preserved over a very long traveled path.

\section{CONCLUSIONS}

We have treated the propagation of a pulse of given initial duration, injected into the fiber by a source with definite temporal coherence properties, by adopting a simple model in which only two modes are considered. This has been performed under the assumption of strong coupling, by means of a deterministic approach, which is able to cover both the resonant and far-from-resonance cases.

Strong coupling is effective in both resonant and nonresonant conditions and affects qualitatively and quantitatively the behavior of dispersion. Far from resonance, dispersion is associated with the breaking up of the initial signal into two distinct pulses proceeding with different velocities, such that the corresponding mutual delay exceeds the temporal broadening of the single pulse. Conversely, this broadening turns out to be the only source of dispersion in the resonant case, where the signal travels as a single pulse.

In both cases, dispersion is drastically reduced with respect to the uncoupled situation, and the main qualitative difference with respect to the weak-coupling regime consists in the linear dependence of pulse dispersion on the traveled length.

\section{ACKNOWLEDGMENTS}

Research supported by the U.S. Army Research Office under Contract No. DAAG29-77-G-0130.

\section{APPENDIX}

According to Eqs. (5) and (39), one has

$$
c_{1}(0, \omega) \propto \int_{-\infty}^{\infty} d t^{\prime} e^{-i \omega t^{\prime}} S\left(t^{\prime}\right) F\left(t^{\prime}\right) e^{i \omega_{0} t^{\prime}},
$$

so that

$$
\begin{aligned}
\left\langle c_{1}(0, \omega) c_{1}^{*}\left(0, \omega^{\prime}\right)\right\rangle_{\mathrm{av}} \propto & \int_{-\infty}^{\infty} d t^{\prime} \int_{-\infty}^{\infty} d t^{\prime \prime} S\left(t^{\prime}\right) S^{*}\left(t^{\prime \prime}\right) \\
& \left.\times e^{-i \Omega t^{\prime}+i \Omega^{\prime} t^{\prime \prime}}\left\langle F\left(t^{\prime}\right) F^{*} t^{\prime \prime}\right)\right\rangle_{\mathrm{av}},
\end{aligned}
$$

where $\Omega=\omega-\omega_{0}$ and $\Omega^{\prime}=\omega^{\prime}-\omega_{0}$. After introducing the time Fourier-transform $\hat{S}(f)$ of the slowly-varying amplitude $S(t)$, one can take advantage of the stationariety of the rapidlyvarying part $F(t)$

$$
\left\langle F\left(t^{\prime}\right) F^{*}\left(t^{\prime \prime}\right)\right\rangle_{\mathrm{av}}=G\left(t^{\prime}-t^{\prime \prime}\right),
$$

thus being able to rewrite Eq. (A2) in the form

$$
\begin{aligned}
\left\langle c_{1}(0, \omega) c_{1}^{*}\left(0, \omega^{\prime}\right)\right\rangle_{\mathrm{av}} & \propto \int_{-\infty}^{\infty} d f \\
& \times \int_{-\infty}^{\infty} d \tau \hat{S}(f) \hat{S}\left(f+\Omega^{\prime}-\Omega\right) G(\tau) e^{-i(\Omega-f) \tau} .
\end{aligned}
$$
has

By recalling that, according to Eqs. (40) and (41), one

$$
S(f) \propto \exp \left(-f^{2} T_{p}^{2} / 4\right)
$$

and

$$
\int_{-\infty}^{\infty} d \tau \exp [-i(\Omega-f) \tau] G(\tau) \propto \exp \left[-(\Omega-f)^{2} T_{c}^{2} / 4\right]
$$

it is possible to perform the integration in Eq. (A4), thus obtaining Eq. (42).

* On leave of absence from Fondazione Ugo Bordoni, Roma, Italy.

'S. D. Personick, "Time Dispersion in Dielectric Waveguides," Bell Syst. Tech. J. 50, 843-859 (1971).

${ }^{2}$ see, also, D. Marcuse, Theory of Dielectric Optical Waveguides, (Academic, New York, 1974).

${ }^{3}$ B. Crosignani, P. Di Porto, and C. H. Papas, "Theory of time-dependent propagation in multimode lightguides," J. Opt. Soc. Am. 67, 1300-1307 (1977).

${ }^{4} \mathrm{~B}$. Crosignani and P. Di Porto, "Coherence of an electromagnetic field propagating in a weakly guiding fiber," J. Appl. Phys. 44, 4616-4617 (1973).

${ }^{5}$ B. Crosignani and P. Di Porto, "Propagation of Coherence and Very High Resolution Measurements in Optical Fibers," in Fibers \& Integrated Optics, S.P.I.E., Vol. 17, (1976).

${ }^{6}$ see, for example, M. Ikeda, Y. Murakami, and K. Kitayami, "Mode scrambler for optical fibers," Appl. Opt. 16, 1045-1049 (1977).

${ }^{7}$ See Ref. 2, p. 101.

${ }^{8}$ See Ref. 2 , p. 105.

${ }^{9}$ See Ref. 2, p. 79

${ }^{10}$ See Ref. 2, p. 212. 